\title{
Current Insight into the Role of Gut Microbiota in Mexican Childhood Obesity
}

\author{
Selvasankar Murugesan ${ }^{1 *}$, Khemlal Nirmalkar ${ }^{1,2}$, Matilde García Espitia ${ }^{3}$, María Luisa Pizano- \\ Zárate $^{4}$, Carlos Hoyo-Vadillo ${ }^{2}$, Jaime Garcia-Mena ${ }^{1}$ \\ ${ }^{1}$ Departamento de Genética y Biología Molecular, Centro de Investigación y de Estudios Avanzados del Instituto Politécnico Nacional. Av IPN 2508 Col \\ Zacatenco, Ciudad de México, 07360, Mexico \\ ${ }^{2}$ Departamento de Farmacología, Centro de Investigación y de Estudios Avanzados del Instituto Politécnico Nacional. Av IPN 2508 Col Zacatenco, \\ Ciudad de México, 07360, Mexico \\ ${ }^{3}$ Escuela Nacional de Medicina y Homeopatía del Instituto Politécnico Nacional, Mexico, Mexico \\ ${ }^{4}$ Departamento de Nutrición, Instituto Nacional de Perinatología, México, Mexico
}

Received: January 14, 2017; Accepted: February 28, 2017; Published: March 14, 2017

*Corresponding author: Selvasankar Murugesan, Departamento de Genética y Biología Molecular, Centro de Investigación y de Estudios Avanzados del Instituto Politécnico Nacional. Av IPN 2508 Col Zacatenco, Ciudad de México, 07360, México, E-mail id: selva@cinvestav.mx

\begin{abstract}
Obesity is an epidemic multifactorial metabolic malady worldwide. This review insights the influence of gut microbiota in developing obesity in Mexican children. High prevalence of childhood obesity in Mexico draws the importance to look for a therapeutic target to control it. Gut microbial disturbances in obese children may have a role in their metabolism. Excessive Short chain fatty acids produced by obese gut microbiota, present an additional energy which causes an imbalance in energy regulation. Thus Manipulating gut microbiota via diverse diet, probiotics and prebiotics treatment can provide a novel approach to treat obesity and other metabolic disorders, including type 2 diabetes and Metabolic syndrome.
\end{abstract}

Keywords: Obesity; Firmicutes; Bacteroidetes; Short chain fatty acids; Mexican children

\section{Introduction}

Obesity is a multifactorial metabolic disease associated with a high risk to develop other chronic diseases and an alarming issue worldwide $[1,2]$. Imbalance between excess caloric intake and too little physical activity are the major factors which contribute to obesity, in addition to genetic and environmental factors. WHO reported that the prevalence of obesity has doubled remarkably worldwide in the last three decades, more than 1.9 billion adults of 18 years and older, were overweight. Among them 600 million were obese [3]. Mexico was placed in eleventh among the most populated country in the world [4]. The rate of obesity in Mexico increased significantly during 2000-2006 period [5]. Mexican National health survey revealed that the population of obesity among school children and adolescents increased from $9.0 \%$ to $14.6 \%$ from the survey 1996 to 2012 which are higher than other Latin American countries such as 7.3\% in Brazil and 3.4\% in Colombia [6,7]. Obesity can affect physical, social and emotional well-being state of a child through clinical complications such as high cholesterol, high blood pressure, asthma, sleep disorders and Nonalcoholic fatty liver diseases. In a population-based study of 5 to 17 year-olds revealed that $70 \%$ of obese youth are more susceptible to cardiovascular disease [8]. Obesity is a process that usually starts in childhood or adolescence, which is a prominent risk factor to other chronic diseases, such as Type 2 Diabetes and coronary disorders [9].

In the recent years, "Gut Microbiota" emerged as a vital field of research interest due to its vast role in human health. Trillions of diverse microorganisms that reside inside the gastrointestinal tract are called as gut microbiota. They can regulate the human physiology directly or indirectly through its metabolites such as Short chain fatty acids (SCFAs), Neurotransmitters and vitamins. An imbalance in the diversity of gut may contribute to several metabolic disorders such as obesity, type 2 diabetes and metabolic syndrome. The aim of this review is to give an insight into the role of gut microbiota as a disposing factor to develop obesity in Mexican children.

\section{Gut Microbiota}

Gut harbors trillions of microorganisms which include bacteria, archaea, yeasts, protozoa, virus and phage. Gut microbiota dispersed in the GI tract as follows, stomach with $10^{1}$ $10^{3} \mathrm{cfu} / \mathrm{ml}$, and duodenum with $10^{1}-10^{3} \mathrm{cfu} / \mathrm{ml}$, ileum with $10^{4}-$ $10^{7} \mathrm{cfu} / \mathrm{ml}$ and colon with $10^{11}-10^{12} \mathrm{cfu} / \mathrm{ml}$ [10]. Among them, bacteria are predominantly abundant in gut microbiota, while Actinobacteria, Bacteroidetes, Firmicutes and Proteobacteria are the most abundant phyla in Gut microbiota [11].

It was assumed that we are exposed to microorganisms at the time of birth and gradually start to be colonized developing a diverse gut microbiota. However, recently, several studies have shown the presence of diverse microbes in placenta, umbilical cord, amniotic fluid, and meconium $[12,13]$. The colonization of gut microbiota can be influenced by several factors such as mode of delivery, breast feeding, diet, antibiotics and life style [14]. The first colonizers are facultative anaerobes, such as Escherichia coli and Streptococcus spp., and obligate anaerobic species colonize as the oxygen levels are low in the gut. In general, the microbial diversity of a child at $\sim 3$ years of age stabilizes to adult-like 
microbiota as shown in a large study of three populations in different geographic locations [15].

Gut microbiota have tremendous potential to affect our physiology, both in health and in disease. They stimulate the maturation of immune system and assist the host by excluding pathogens. Many Studies have exposed the intestinal microbiota capability to manipulate host physiology to benefit both microbe and host [16]. The gut microbial communities and their metabolic activity are influenced by a several factors including the diet and health status of the host [17].

Since most of the gut microbes are not cultivable, culture independent techniques such as DGGE, RT-PCR and High throughput sequencing are the suitable tools to explore microbial diversity. DGGE is a effective but laborious and time taking technique. RT-PCR is effective but it is not appropriate for unexplored microorganisms. High throughput sequencing techniques on the other hand are currently lower cost and more effective to explore microbial diversity. 16S rDNA based sequencing of bacterial hypervariable regions or metagenomic sequencing is the immensely used methods in the field of microbiota. The $16 \mathrm{~S}$ rDNA based technique helps to explore the diversity and metagenome is used to explore marker genes, metabolic route and enterotype patterns among different clinical conditions or experimental systems.

\section{Role of Gut Microbiota}

Gut microbiota conduct essential functions such as maintenance of gut, immune functions and digestion of nutrients by a symbiotic relationship with human host body [18] Undigested carbohydrate fibers are fermented by gut microbiota and produces principal metabolites such as SCFA(acetic acid$65 \%$, propionic acid-25\% and butyric acid-15\%), vitamins, it degrades many dietary toxins and carcinogens, protects host from pathogens, maturation of immune system, Intestinal permeability, inflammation control and bile acid metabolism (Figure 1). Dysbiosis of gut microbiota, have been related with many diseases such as cancer, Rheumatoid arthritis including cardiovascular diseases, obesity, diabetes and metabolic syndrome. Role of gut microbiota also had been reported in cognitive disorders like Alzheimer and Parkinson's diseases liver or even brain diseases $[19,20]$.

\section{Gut microbial Metabolites}

The gut microbiota can produce extremely diverse metabolites from anaerobic fermentation of exogenously undigested dietary fibers which reach the colon and endogenous compounds released from the microorganisms harbor in the host. These metabolites act as intermediators between the gut microbiota and the host to regulate many immunological functions and diseases control. SCFAs, TMAO (Trimethyl amine oxide), Polyphenols, Bile acids, Neurotransmitters such as serotonin, Dopamine, acetylcholine, Gamma amino butyric acid (GABA),B-vitamins, Polyamines are those well-known metabolites due to their impact on host physiology[21]. Among them, SCFAs play a vital role in host energy metabolism.

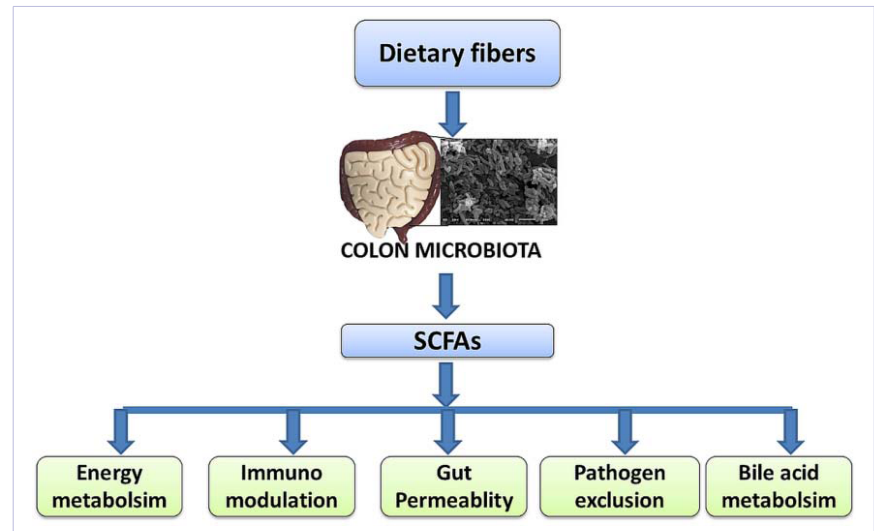

Figure 1: Role of Colon microbiota in human physiology.

\section{Short Chain Fatty Acids (SCFA)}

Soluble dietary fibers such as fructans, pectin, inulin and xylans and resistant starch can be actively saccharified by commensal microbiota in the distal colon of the host and produce biologically active SCFAs (acetate, propionate and butyrate). SCFAs are the main energy source (up to $70 \%$ ) for the intestinal epithelial cells [22]. Firmicutes and Bacteroidetes are the main contributors to produce SCFAs in gut microbiota. Among these bacteria, Bacteroidesspp., Faecalibacterium spp., Bifidobacterium spp., Clostridium spp., Eubacterium spp, Lactobacillus spp. And Ruminococcus spp. has been reported as best producers [23].

Microbe-generated acetate has a number of physiological functions. Acetic acid, is a substrate for cholesterol synthesis [24], as well as a suppressor of appetite through a central hypothalamic mechanism [25].Propionic acid is a precursor for protein synthesis, gluconeogenesis, and liponeogenesis in the liver [26]. Acetate and propionate also induces adipogenicity through GPCR43 [27]. The colonic epithelium receives about 70 $\%$ of its energy from SCFAs, mainly from butyric acid [28]. Butyric acid is a Histone deacetylase inhibitor through which it regulates the gene expression and cell fate [29].

SCFAs are also involved in the improvement of cognition and behavior through the microbiota-gut-brain axis [30]. In neurodegenerative diseases such as Alzheimer's, Parkinson's and Huntington's diseases, there is a reported deficiency in glucose uptake, the main fuel source for astrocytes [31]. However, ketone bodies of SCFA origin such as $\beta$-hydroxy butyrate, acetoacetate, acetone and lactate can also function as substitute energy source in cognitive disorders. SCFAs moderate the inflammatory function by triggering the production of mediators like cytokines and Nitrogen Oxide (NO) by macrophages [32]. Among SCFA, butyrate inhibits de Histone Deacetylase (HDAC), and increases the availability of Histone acetyl Transferase (HAT) which increases histone acetylation. This inhibition has been reported that improves neuroprotection/regeneration in both in vitro and in vivo models of neurodegenerative diseases [33]. SCFAs may also influence innate immune cells in the brain and central nervous system. 


\section{Gut Microbiota and Host Metabolism}

Gut microbiota provides an additional metabolic energy through undigested carbohydrate fibers. The Main metabolic products, SCFAs can be utilized for lipid or glucose denovo synthesis [34]. Alteration in the levels of SCFAs in obesity might be due to dysbiosis in the colon microbiota. So it is essential to explore the distal colon community members and SCFAs level to comprehend its role in development of obesity. This complex microbial system has the higher metabolic capacity and plays a vital role in regulating several processes in host intestine [18].

The basis that lead to the development of obesity, shows that in addition to the genetic component of the human genome; in many cases there is a clear influence of the human microbiome [35]. The microbiome is the full set of genes in the genomes of all microbes that live in the human body, and whose expression has influence on its systemic function [36]. Recent reports in mice model have shown that overweight and obesity are associated with a particular type of bacteria that inhabit the digestive tract; other studies in adult humans, have shown that variations in the relative abundance of two phyla Firmicutes and Bacteriodetes, are related to the condition of accumulation of body fat [37].

The short-chain fatty acids (SCFAs) have been used traditionally as a therapy for colitis and ulcerative colitis, due to their anti-inflammatory effect [38]. It is presumed that inhibition of the NFKB factor is involved in its mechanism of action; thus, these metabolites would be related with signal transduction pathways with influence in systemic inflammation state $[39,40]$.

\section{Gut Microbiota and Obesity}

The gut microbiota involves in the fermentation of undigested polysaccharides to easily absorbable monosaccharides, and lipoprotein lipase activation on the villous epithelium, playing a role in nutrient acquisition and energy regulation by the host $[41,42]$. It has been suggested that gut microbiota contributes to obesity, by increasing energy harvesting from diet, and modulating through its metabolites, via hostlipid metabolic pathways and energy regulating homoeostasis [43]. To date, the reported main metabolic products of colon microbiota are acetic, propionic, and butyric acids. Alteration in the levels of SCFAs in obesity, might be associated to bacterial dysbiosis in the gut; making it essential to explore the diversity of bacterial communities and the SCFAs level to realize its role in the development of this disease (Figure 2).

It has been reported that difference in composition of gut microbiota with more abundant Firmicutes in obese than lean subjects which lead to the disruption in energy acquisition and regulation [44]. It has been demonstrated that overweight and obese adults from Netherlands have elevated fecal SCFA concentrations and Firmicutes abundance than their lean counterparts. It suggested that these obese individuals produce more colonic SCFA, indicating an increased microbial energy harvest [45]. Alteration in the levels of SCFAs in obesity might be due to dysbiosis in the colon microbiota.

In a study on obese Finnish children showed lower Bifidobacterium spp. and higher Staphylococus aureus abundances

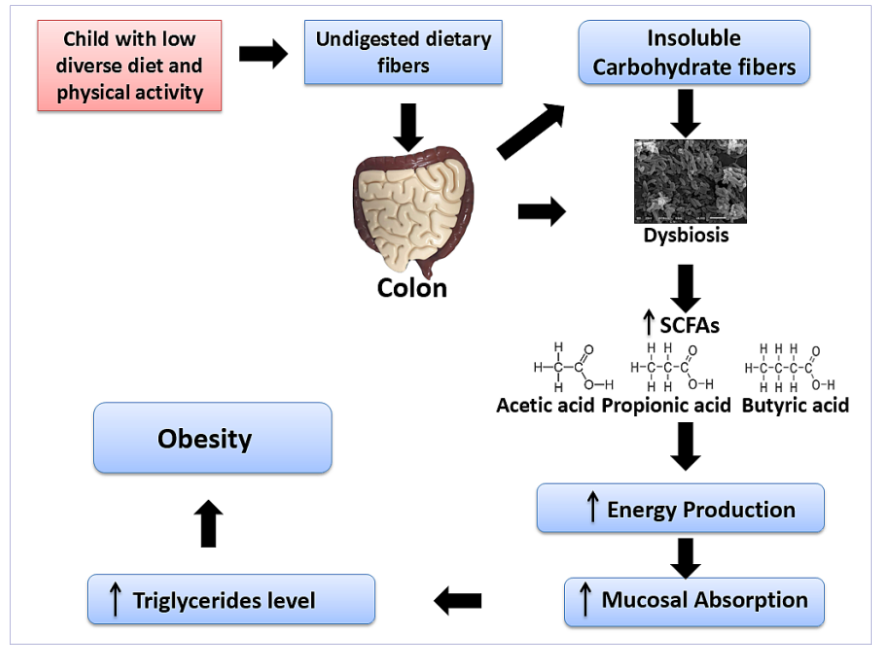

Figure 2: Influence of SCFAs in development of Obesity.

in obese children compared with normal weight children [46]. Another study on Obese children from Belgium revealed that obese children had a higher ratio of Firmicutes/Bacteroidetesas well as more abundant Lactobacillus spp. and Staphylococcus spp. which was positively correlated with inflammatory markers such as C-reactive protein and energy intake, respectively [47].

Only few literatures disclose the role of microbiota associated with obesity in Mexican children. In one important report, the fecal SCFA concentrations was lower in overweight and obese Mexican children with respect to normal children and was associated to an imbalance in the abundance of Roseburia spp., Blautia spp., Coprococcus spp., Faecalibacterium spp. and families like Lachnospiraceae which belongs to the phylum Firmicutes. The elevated triglycerides concentration in obese children might be the consequence of increased in mucosal absorption of SCFA [48]. This condition will enhance the development of metabolic disorder obesity along with lower physical activity. In has been also suggested a possible association of endothelial dysfunction with an alteration of gut microbiota in obese children [49]. Thus, it is considered that the gut microbiota plays an important role in weight regulation and may even be partly responsible for the development of obesity.

\section{Conclusion}

These collective evidences strongly propose that the gut microbiota play a significant role in regulating the energy balance and weight in children and may influence the development and progression of obesity in addition to lack of physical activity and other genetic factors. Manipulating gut microbiota through diverse diet, probiotics and prebiotics treatment can give a novel approach to treat obesity and other metabolic disorders, including type 2 diabetes and Metabolic syndrome.

\section{Acknowledgment}

This work was funded by Cinvestav; CONACyT 163235 INFR-2011-01; and FONSEC SS/IMSS/ISSSTE-CONACYT-233361 granted to JGM. We thank a Postdoctoral Fellowship from FONSEC SS/IMSS/ISSSTE-CONACYT-233361 granted to SM. 


\section{References}

1. Waalen J. The Genetics of Human Obesity. TranslRes. 2014;164(4):293301.

2. James WPT. The Epidemiology of Obesity: The Size of the Problem J Intern Med. 2008;263(4):336-352. doi: 10.1111/j.13652796.2008.01922

3. World Health Organization. Obesity and Overweight. 2016.

4. United Nations. Trends world population. 2015. Available from: http:// www.un.org/en/development/desa/population/publications/ trends/wpp2012.shtml

5. Barquera S, Campos-Nonato I, Hearnandes-Barrera L, Flores M, Durazo-Arvizu R, Kanter R, et al. Obesity and central adiposity in Mexican adults: results from the Mexican National Health and Nutrition Survey 2006. Salud PublicaMex. 2009; 51:S595-S603.

6. ENSANUT. Encuesta Nacional de Salud y Nutrición 2016. Available from: http://ensanut.insp.mx/informes/ ENSANUT2012ResultadosNacionales.pdf

7. Rivera JÁ, de Cossío TG, Pedraza LS, Aburto TC, Sa'nchez TG, Martorell R. Childhood and adolescent overweight and obesity in Latin America: a systematic review. Lancet Diabetes Endocrinol. 2014;2(4):321-332. doi: 10.1016/S2213-8587(13)70173-6.

8. Freedman DS, Zuguo M, Srinivasan SR, Berenson GS, Dietz WH. Cardiovascular risk factors and excess adiposity among overweight children and adolescents: the Bogalusa Heart Study. J Pediatr. 2007; $150(1): 12-17$.

9. Wild SH, Byrne CD. Risk Factors for Diabetes and Coronary Heart Disease Dyslipidaemia. BMJ. 2006;333:1009-1110.

10. O'Hara AM, Shanahan F. The gut flora as a forgotten organ. EMBO Rep. 2006;7(7):688-693. doi: 10.1038/sj.embor.7400731.

11. Tremaroli V, Bäckhed F. Functional interactions between the gut microbiota and host metabolism. Nature. 2012;489:242-249. doi: $10.1038 /$ nature 11552 .

12. Jiménez E, Fernández L, Marín ML, Martín R, Odriozola JM, NuenoPalop $\mathrm{C}$, et al. Isolation of commensal bacteria from umbilical cord blood of healthy neonates born by cesarean section. CurrMicrobiol 2005;51:270-274.

13. Moles L, Gómez M, Heilig H, Bustos G, Fuentes S, de Vos W, et al Bacterial diversity in meconium of preterm neonates and evolution of their fecal microbiota during the first month of life. PLoS ONE. 2013;8(6):e66986. doi:10.1371/journal.pone.0066986.

14. Jandhyala SM, Talukdar R, Subramanyam C, Vuyyuru H, Sasikala M, Reddy DN. Role of the normal gut microbiota. World Journal of Gastroenterology: WJG. 2015;21(29):8787-8803. doi:10.3748/wjg. v21.i29.8787

15. Yatsunenko T, Rey FE, Manary MJ, Trehan I, Dominguez-Bello MG, Contreras $\mathrm{M}$, et al. Human gut microbiome viewed across age and geography. Nature. 2012;486:222-227. Doi:10.1038/nature11053.

16. Kelly D, Campbell JI, King TP, Grant G, Jansson EA, Coutts AG, et al. Commensal anaerobic gut bacteria attenuate inflammation by regulating nuclearcytoplasmic shuttling of PPAR-gamma and RelA. Nat Immunol. 2004;5(1):104-12.

17. De Filippo C, Cavalieri D, Di Paola M, Ramazzotti M, Poullet JB, Massart S, et al. Impact of diet in shaping gut microbiota revealed by a comparative study in children from Europe and rural Africa. Proc Natl AcadSci U S A. 2010;107(33):14691-14696. doi: 10.1073/ pnas.1005963107.
18. Backhed F, Ley RE, Sonnenburg JL, Peterson DA, Gordon JI. Host-bacterial mutualism in the human intestine. Science. 2005;307:(5717):1915-1920. DOI:10.1126/science.1104816

19. Duca F, Gerard P, Covasa M, Lepage P. Metabolic interplay between gut bacteria and their host. Front Horm Res. 2014;42:73-82. doi:10.1159/000358315.

20. Mayer EA, Tillisch K, Gupta A. Gut/brain axis and the microbiota. J Clin Invest. 2014;125(3):926-938. doi: 10.1172/JCI76304.

21. Rooks MG, Garrett WS. Gut microbiota, metabolites and host immunity. Nat Rev Immunol. 2016;16(6):341-52. doi: 10.1038/nri.2016.42.

22. Cummings JH. Fermentation in the human large intestine: evidence and implications for health. Lancet. 1983;1(8335):1206-1209.

23. Louis P, Young P, Holtrop G, Flint HJ. Diversity of human colonic butyrate-producing bacteria revealed by analysis of the butyryl-CoA:acetate CoA-transferase gene. Environ Microbiol. 2010;12(2):304-314. doi: 10.1111/j.1462-2920.2009.02066.x.

24. Vogt JA, Wolever TMS. Fecal acetate is inversely related to acetate absorption from the human rectum and distal colon. J Nutr. 2003;133(10):3145-3148.

25. Frost G, Sleeth ML, Sahuri-Arisoylu M, Lizarbe B, Cerdan S, Brody L et al. The short-chain fatty acid acetate reduces appetite via a central homeostatic mechanism. Nat Commun. 2014;5:3611. doi:10.1038/ ncomms4611.

26. Kotzampassi K, Giamarellos-Bourboulis EJ, Stavrou G. Obesity as a consequence of gut bacteria and diet interactions. ISRN Obes. 2014;2014:651895. doi:10.1155/2014/651895.

27. Hong YH, Nishimura Y, Hishikawa D, Tsuzuki H, Miyahara H, Gotoh $\mathrm{C}$, et al. Acetate and propionate short chain fatty acids stimulate adipogenesis via GPCR43. Endocrinology. 2005;146(12):5092-5099.

28. Scheppach W. Effects of short chain fatty acids on gut morphology and function. Gut. 1994;35(1):S35-S38.

29. Canani RB, Costanzo MD, Leone L, Pedata M, Meli R, Calignano A. Potential beneficial effects of butyrate in intestinal and extraintestinal diseases. World Journal of Gastroenterology : WJG. 2011;17(12):15191528. doi: 10.3748/wjg.v17.i12. 1519.

30. Stilling RM, Dinan TG, Cryan JF. Microbial genes, brain \&behaviourepigenetic regulation of the gut-brain axis. Genes Brain Behav. 2014;13(1):69-86. doi: 10.1111/gbb.12109.

31. Beltrán FA, Acuña AI, Miró MP, Castro MA. Brain Energy Metabolism in Health and Disease. In:Carlos M. Contreras, editor. Neuroscience Dealing With Frontiers. $1^{\text {st }}$ Ed. InTech Open; 2012:331-362.

32. Vinolo MAR, Rodrigues HG, Nachbar RT, Curi R. Regulation of Inflammation by Short Chain Fatty Acids. Nutrients. 2011;3(10):858876. doi: 10.3390/nu3100858.

33. Govindarajan N, Agis-Balboa RC, Walter J, Sananbenesi F, Fischer A. Sodium butyrate improves memory function in an Alzheimer's disease mouse model when administered at an advanced stage of disease progression. J Alzheimers Dis. 2011;26(1):187-197. DOI: 10.3233/ JAD-2011-110080

34. Palmer C, Bik EM, DiGiulio DB, Relman DA, Brown PO. Development of the Human Infant Intestinal Microbiota. PLoS Biol. 2007;5(7):e177.

35. Devaraj S, Hemarajata P, Versalovic J. The human gut microbiome and body metabolism: implications for obesity and diabetes. Clin Chem. 2013;59(4):617-628. doi: 10.1373/clinchem.2012.187617. 
36. Ursell LK, Metcalf JL, Parfrey LW, Knight R. Defining the human microbiome. Nutr Rev. 2012;70 (Suppl 1):S38-S44. doi: 10.1111/j.1753-4887.2012.00493.x.

37. Ley RE. Obesity and the Human Microbiome. CurrOpinGastroenterol 2010;26(1):5-11.

38. Whelan K, Judd PA, Preedy VR, Taylor MA. Enteral Feeding: The Effect on Faecal Output, the Faecal Microflora and SCFA Concentrations. Proc Nutr Soc. 2004;63(1):105-113.

39. Segain J, De la Bleitere DR, Boureille A, Leray V, Gervois N, Rosales $C$, et al. Butyrate inhibits inflammatory responses through NFkB Inhibition: Implications for Crohn's disease. Gut. 2000;47(3):397403.

40. Dengler F, Rackwitz R, Benesch F, Pfannkuche H, Gabel G. Both butyrate incubation and hypoxia upregulate genes involved in the ruminal transport of SCFA and their metabolites. J AnimPhysiolAnimNutr (Berl). 2015;99(2):379-390. doi: 10.1111/jpn.12201.

41. Bäckhed F, Ding H, Wang T, Hooper LV, Koh GY, Nagy A, et al. The Gut Microbiota as an Environmental Factor That Regulates Fat Storage. Proc Natl AcadSci U S A. 2004;101(44):15718-15723.

42. DiBaise JK, Zhang H, Crowell MD, Krajmalnik-Brown R, Decker GA, Rittmann BE. Gut Microbiota and Its Possible Relationship with Obesity. Mayo Clin Proc. 2008;83(4):460-469. doi: 10.4065/83.4.460.
43. Flint HJ. Obesity and the Gut Microbiota. J ClinGastroenterol. 2011;45(Suppl):S128-132. doi: 10.1097/MCG.0b013e31821f44c4.

44. Ley R, Turnbaugh P, Klein S, Gordon J. Microbial ecology: human gut microbes associated with obesity. Nature. 2006;444:1022-1023.

45. Schwiertz A, Taras D, Schäfer K, Beijer S, Bos NA, Donus C et al. Microbiota and SCFA in lean and overweight healthy subjects. Obesity. 2010;18(1):190-195. doi: 10.1038/oby.2009.167.

46. Kalliomaki M, Collado M C, Salminen S, Isolauri E. Early differences in fecal microbiota composition in children may predict overweight. Amer J ClinNutr. 2008;87:534-538.

47. Bervoets L, Van Hoorenbeeck K, Kortleven I, Van Noten C, Hens $\mathrm{N}$, Goossens $\mathrm{H}$, et al. Differences in gut microbiota composition between obese and lean children: a cross-sectional study. Gut Pathog. 2013;5:10. doi: 10.1186/1757-4749-5-10.

48. Murugesan S, Ulloa-Martínez M, Martínez-Rojano H, Galván-Rodríguez FM, Miranda-Brito C, Romano MC et al. Study of the diversity and short-chain fatty acids production by the bacterial community in overweight and obese Mexican children. Eur J ClinMicrobiol Infect Dis. 2015;34:1337-1346. doi: 10.1007/s10096-015-2355-4.

49. Nirmalkar K, Murugesan S, Pizano-Zárate ML, Romero-Figueroa MS, Hoyo-Vadillo C, Garcia-Mena J. Endothelial Dysfunction in Mexican Obese Children, is there A Role of the Gut Microbiota? Obes Control Ther. 2016;3(1):1-4. 